Copyright (C 2014 IEEE. Personal use of this material is permitted. Permission from IEEE must be obtained for all other uses, in any current or future media, including reprinting/republishing this material for advertising or promotional purposes, creating new collective works, for resale or redistribution to servers or lists, or reuse of any copyrighted component of this work in other works. 


\title{
A Fuzzy Control Based Self-Optimizing PID Model for Autonomous Car Following on Highway
}

\author{
Qing Wang, Shou-zhi $\mathrm{Xu}^{*}$ \\ College of Computer and Information technology \\ China Three Gorges University \\ Yi Chang, China \\ E-mail: xsz@ctgu.edu.cn \\ Honglei Xu \\ Department of Mathematics of Statistics \\ Curtin University, \\ Perth, WA, Australia
}

\begin{abstract}
Controlling real-time distances between vehicles is extremely significant for keeping safe driving on highway. At the same time, status of traffic stream, whole time delay and errors in controlling also have impacts on the control at a high driving speed. In this paper, a self-optimizing PID model based on fuzzy control is proposed for assisting a car to follow another one at a safe distance. The proposed model is established on a wireless sensor network sharing information for perceiving different status of traffic stream. In the designed experiment, the fuzzy PID controller is able to adapt nonlinear and time-varying features of behaviors in traffic stream. Comparing with general PID controllers, the fuzzy PID controller has a better performance on dynamic behaviors. According to comparison with the effects of controlling in different conditions, simulation results show that the proposed model is able to adjust and control the follower's behaviors in different situations on highway.
\end{abstract} PID

Keywords-Vehicles on highway; Car-Following model; Fuzzy

\section{REVIEW OF CAR-FOLLOWING TECHNOLOGIES}

Transportation in highway is one of the most important ways, which undertakes $70 \%$ of passenger transport and $40 \%$ of freightage [1]. Recent surveys from the department of transport point out that the accident rate in highway is four times higher than ordinary roads [2]. Researches on the highway vehicle state are of great significance for traffic safety.

A car-following model is one of the most important models of driving behaviors in a transportation system. The general model is theoretically based on driver's psychological uncertainties and inconsistent activities [3], such as traditional GM (General Model) model, Helly linear model. A distance between two vehicles is a key factor to affect the follower' status traveling on highway [4]. In recent years, a large number of theoretical studies on the distance have been conducted including traffic warning based on the safe distance in driving [5], distribution distances analysis of highway vehicles [6], and safe distance's impact on steady car's following state [7-9]. The car-following is a very complex process, and current studies of the behaviors are mostly focusing on the investigation of the theoretical system. However, in the application of these models, the nonlinear factors, such as speed changes and driver's subjective behaviors, affect the measurement of the real distance and the determination of the safe distance, which will limit the application of the obtained general control methods. On the other hand, fuzzy control is a control method using fuzzy logic and mathematical analysis theory, which does not rely on the mathematical model of the controlled object. The method can establish the controlling rules based on the practical application, and it is also capable of adapting to the nonlinear and time-varying system [10, 11$]$. Comparing with the traditional control methods, the selfoptimizing model based on fuzzy control can improve dynamic performance effectively and achieve better controlling effects [12-14].

Therefore, using fuzzy control theory, we establish a relationship among three PID controller parameters $k_{p}, k_{i}, k_{d}$, the deviation of the difference of real-time distance and safe distance, and the deviation of the change rate. The proposed method combines with the actual needs of highway vehicles and builds fuzzy control rules to achieve self-optimizing adjusting parameters. The simulation results show that the selfoptimizing PID model based on fuzzy control can adapt to the dynamic characteristics of car-following behavior and improve the applicability for highway car-following safety.

\section{AN IMPROVED CAR-FOLLOWING MODEL}

\section{A. Foundation of Designed Model}

The designed car-following model is based on the information spreading in traffic network. Vehicles are all abstracted as the nodes of the network and they communicate each other wirelessly, as shown in Fig. 1. Many kinds of sensors are equipped for each node to achieve information 
during driving, such as speed, acceleration, and orientation sensors.

In the traffic network, the wireless communication among the nodes can achieves the sharing information of each one in the network. It is fundamental to compute the average speed of the flow to get the status to determine a safe distance, which contributes to keep safe driving in highway.

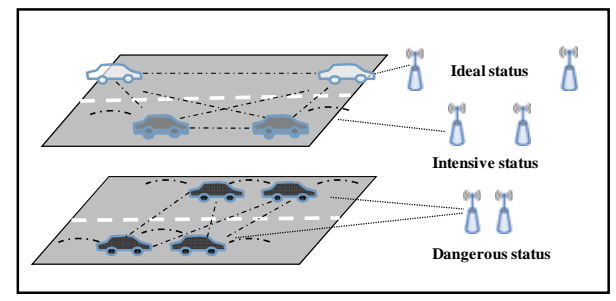

Figure 1. The abstraction of vehicles in traffic flow.

\section{B. Status of Vehicles in Driving}

According to the three-phase traffic flow theory, the status of vehicles will be generally divided into three types: free flow, synchronized flow, and wide moving jam. This paper will define three kinds of states based on traffic flow theory, called dangerous status, intensive status and ideal status. The safe distance will be different in different cases. As shown in Fig. 2, black, gray, white cars represent the ideal, the intensive and the dangerous status, respectively. Furthermore, the average speed of the flow is an essential factor to determine the range of the current vehicle safety distance in the corresponding status.

In our simulation, the current average speed is $100 \mathrm{~km} / \mathrm{s}$ by computing the obtained data. According to the driving rules in highway, the corresponding safe distance should be more than $100 \mathrm{~m}$, at the same time the vehicles driving in the ideal status; when the distance is in $65 \sim 100 \mathrm{~m}$, the density of vehicles is increasing to be in the intensive status; When $d$ is much less than $65 \mathrm{~m}$, the object is in the danger and the driver must adjust in time. The process ensures the safety and improves the effective driving. As mentioned above, the driving distance between vehicles in the different conditions should be adjusted to be in the corresponding safe range.

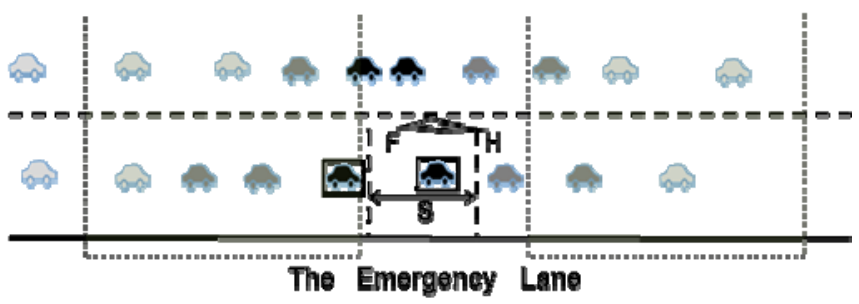

Figure 2. The driving of vehicles in highway.

The research on safe following is conducted as follows: Assume that two vehicles are driving in highway. They are F and $\mathrm{H}$ in Fig. 2, where $\mathrm{H}$ is the one ahead. In this case, $\mathrm{F}$ judges its own status in the flow by analyzing the information from the network, identifies the safe driving range, and adjusts the distance between $\mathrm{H}$ according to the current driving speed. (1) If $F$ is in the ideal status, to ensure a comfortable driving is an important goal, so $\mathrm{F}$ can adjust the distance slowly, and ultimately keep in the safe range; (2) If the F drives in the intensive status, the driver should be cautious for adjusting the distance, and stabilize at a safe distance in time; (3) If the vehicle is in the dangerous status, $\mathrm{F}$ must be controlled in the least error and delay. The process analysis is shown in Fig. 3.

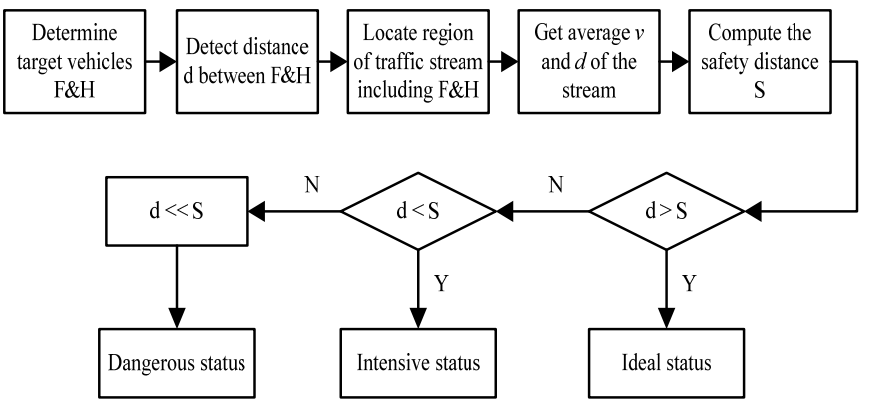

Figure 3. The analysis of vehicle statuses.

As shown in Fig. 2, the region of interest is in the dangerous state, namely the distance between $\mathrm{F}$ and $\mathrm{H}$ is far less than the current safe distance. Under the circumstance, the driver must be adjusted rapidly.

\section{Mathematical Model}

Traffic stream in highway can be regarded as a queue with multi-channel and same direction. A highway includes a driving lane, a passing lane and an emergency lane, as in Fig. 2. Vehicles are generally running in the driving lane. Through analyzing the characteristics of the vehicles in the driving lane, this paper will propose the following nonlinear continuous model in Fig. 4.

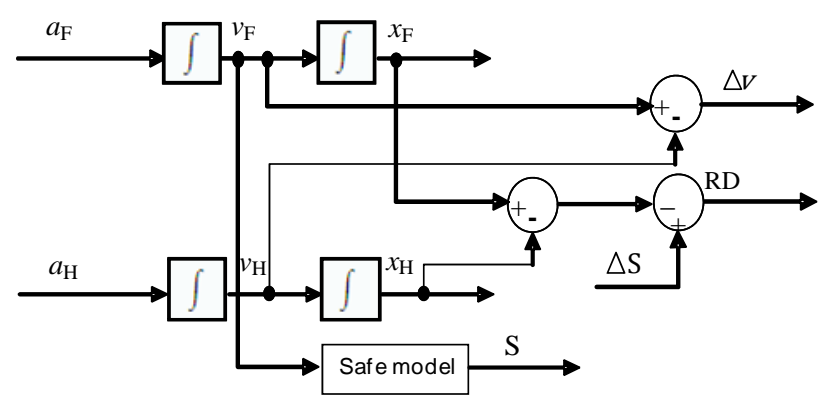

Figure 4. The analysis of the following behavior.

$a_{\mathrm{F}}, a_{\mathrm{H}}, v_{\mathrm{F}}, v_{\mathrm{H}}, x_{\mathrm{F}}, x_{\mathrm{H}}$, represent the acceleration, speed and displacement of $\mathrm{F}$ and $\mathrm{H}$, respectively; $\Delta v$ is the relative speed between $\mathrm{F}$ and $\mathrm{H}$; $\mathrm{S}$ is the safe distance computing from safe model based on the current speed of $\mathrm{F}$; RD is the real distance of two cars, $\Delta S$ is the initial distance of them. According to 
the driving status of $\mathrm{F}$ and $\mathrm{H}$, the space state equation is proposed in (1).

$$
\left\{\begin{array}{l}
\dot{\bar{x}}=\mathrm{A} \bar{x}+\mathrm{B} u \\
y=\mathrm{C} \bar{x}+\mathrm{D} u
\end{array}\right.
$$

where A, B, C, D are the parameter matrixes; $x, \dot{\bar{x}}$ is the state matrix, [ $x_{1}, x_{2}, x_{3}, x_{4}$ ], [ $\left.\dot{\bar{x}}_{1}, \dot{\bar{x}}_{2}, \dot{\bar{x}}_{3}, \dot{\bar{x}}_{4}\right] ; x_{1}, x_{2}$ are the speed of $\mathrm{F}$ and $\mathrm{H} ;{ }^{x_{2}},{ }^{x_{4}}$ are the displacements of $\mathrm{F}, \mathrm{H}$; $u$ is input matrix [ ${ }^{u_{1}},{ }_{2}$ ], and ${ }^{u_{2}},{ }^{u_{1}}$ represent the acceleration of $\mathrm{F}, \mathrm{H}$. Through the analysis of the following behavior, the parameter matrixes are respectively determined,

$$
\mathrm{A}=\left[\begin{array}{llll}
0 & 0 & 0 & 0 \\
1 & 0 & 0 & 0 \\
0 & 0 & 0 & 0 \\
0 & 0 & 0 & 0
\end{array}\right], \mathrm{B}=\left[\begin{array}{ll}
1 & 0 \\
0 & 0 \\
0 & 1 \\
0 & 0
\end{array}\right], \mathrm{C}=\left[\begin{array}{llll}
0 & -1 & 0 & 1
\end{array}\right], \mathrm{D}=[0,0] \text {. }
$$

In the driving process of $\mathrm{F}$ and $\mathrm{H}$, the status can be learned in real time, through the speed sensor, acceleration sensor, and other sensors.

\section{FUZZY PID SYSTEM}

In the application of control model, since the varying factors, such as the driving speed, safe distance, environmental conditions, the driving status of the vehicle will change all the time. In this case, the general PID controller is difficult to obtain an efficient control. Fuzzy control is defined by fuzzy set theory, fuzzy mathematics, fuzzy language representation and inference rules of fuzzy logic, which does not rely on the mathematical model of the controlled object. This application can ensure dynamic response and improve accuracy of controlling in system. Therefore, this paper designs a fuzzy self-optimization model on the basis of the vehicle driving rules and PID control.

The designed car-following system based on fuzzy selfoptimizing PID control is divided into four parts: safe distance adjustment, fuzzy PID control, model of the controlled objects and feedback. (1) According to the average speed of the traffic stream included the target vehicles, the system can learn the driving status of vehicle and compute the real-time safe distance. (2) Fuzzy PID control is the combination of fuzzy controller and PID controller. In the control model, the feedback value deviation e and the change rate of deviation ec are inputs of fuzzy PID controller. Through the fuzzy PID controller, parameters $\mathrm{k}_{\mathrm{p}}, \mathrm{k}_{\mathrm{i}}, \mathrm{k}_{\mathrm{d}}$ are dynamically optimized; (3) The controlled objects are information of two vehicles, that one is following the other, including the displacement and speed of the two vehicles and acceleration of the following; (4) Fuzzy PID system inputs the real-time acceleration of the vehicle into the control model for adjusting. Finally, output of the system, relative distance between the vehicles, acts as a feedback to affect the system. The structure of the system is shown in Fig. 5.

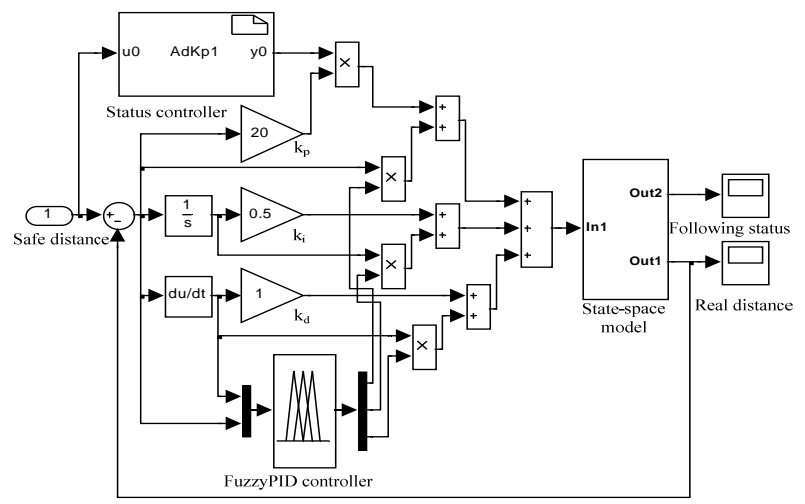

Figure 5. The model of fuzzy PID system simulation

The relationship among three parameters of controller, the deviation e and the change rate of the deviation ec are the key factors to achieve self-optimization in control. For getting better controlling effect, e and ec act as the inputs of system, and three parameters are adjusted by the fuzzy control rules in tables.

\begin{tabular}{|c|c|c|c|c|c|c|c|}
\hline \multirow{2}{*}{ ec } & \multicolumn{7}{|c|}{ Seven dimensions of $k_{p}$} \\
\hline & NB & $N M$ & NS & $Z O$ & $P S$ & $P M$ & $\overrightarrow{P B}$ \\
\hline NB & PB & PB & PB & PB & PS & $\mathrm{zO}$ & NS \\
\hline NM & PB & PB & PM & PM & $\mathrm{ZO}$ & NS & NM \\
\hline NS & PB & PM & PM & PS & NS & NM & NB \\
\hline $\mathrm{ZO}$ & $\mathrm{ZO}$ & 20 & $\mathrm{zO}$ & $\mathrm{ZO}$ & $\mathrm{zO}$ & $\mathrm{zO}$ & $\mathrm{zO}$ \\
\hline PS & NB & NM & NS & PS & PM & PM & PB \\
\hline PM & NM & NS & $\mathrm{zO}$ & PM & PB & $\mathrm{PB}$ & PB \\
\hline PB & NS & $\mathrm{zO}$ & PS & PB & PB & PB & PB \\
\hline
\end{tabular}

TABLE I. The Fuzzy CONTROL Rules of PARAMETERS KP.

\begin{tabular}{|c|c|c|c|c|c|c|c|}
\hline \multirow{2}{*}{ ec } & \multicolumn{7}{|c|}{ Seven dimensions of $\mathbf{k}_{\mathbf{i}}$. } \\
\hline & $a$ & $N M$ & $N S$ & $\mathrm{ZO}$ & $P S$ & $P M$ & $P B$ \\
\hline NB & PB & PB & PB & PB & NS & NM & NB \\
\hline NM & PB & PB & PM & $\mathrm{PM}$ & NM & NB & NB \\
\hline NS & PB & PM & PS & PS & NB & NB & NB \\
\hline $\mathrm{ZO}$ & $\mathrm{ZO}$ & $\mathrm{ZO}$ & $\mathrm{ZO}$ & $\mathrm{ZO}$ & $\mathrm{ZO}$ & $\mathrm{ZO}$ & $\mathrm{ZO}$ \\
\hline PS & NB & NB & NB & PS & PS & PM & $\mathrm{PB}$ \\
\hline $\mathrm{PM}$ & NB & NB & $\mathrm{NM}$ & $\mathrm{PM}$ & PM & PB & $\mathrm{PB}$ \\
\hline PB & NB & NM & NS & PB & PB & PB & PB \\
\hline
\end{tabular}

TABLE II. The Fuzzy CONTROL RUlES OF PARAMETERS KI. 
TABLE III. THE FUZZY CONTROL RULES OF PARAMETERS KD.

\begin{tabular}{c|l|l|l|l|l|l|l}
\hline \multirow{2}{*}{$\mathbf{e}$} & \multicolumn{7}{c}{ Seven dimensions of $\mathbf{k}_{\mathbf{d}}$} \\
\cline { 2 - 8 } & $\mathbf{N B}$ & $\mathbf{N M}$ & NS & $\mathbf{Z O}$ & $\mathbf{P S}$ & $\mathbf{P M}$ & $\mathbf{P B}$ \\
\hline $\mathrm{NB}$ & $\mathrm{PB}$ & $\mathrm{PB}$ & $\mathrm{PB}$ & $\mathrm{NB}$ & $\mathrm{NB}$ & $\mathrm{NM}$ & $\mathrm{NS}$ \\
$\mathrm{NM}$ & $\mathrm{PB}$ & $\mathrm{PB}$ & $\mathrm{PM}$ & $\mathrm{NM}$ & $\mathrm{ZO}$ & $\mathrm{PS}$ & $\mathrm{PM}$ \\
$\mathrm{NS}$ & $\mathrm{PB}$ & $\mathrm{PM}$ & $\mathrm{PM}$ & $\mathrm{NS}$ & $\mathrm{PM}$ & $\mathrm{PB}$ & $\mathrm{PB}$ \\
$\mathrm{ZO}$ & $\mathrm{ZO}$ & $\mathrm{ZO}$ & $\mathrm{ZO}$ & $\mathrm{ZO}$ & $\mathrm{ZO}$ & $\mathrm{ZO}$ & $\mathrm{ZO}$ \\
$\mathrm{PS}$ & $\mathrm{PB}$ & $\mathrm{PB}$ & $\mathrm{PM}$ & $\mathrm{NS}$ & $\mathrm{PM}$ & $\mathrm{PM}$ & $\mathrm{PB}$ \\
$\mathrm{PM}$ & $\mathrm{PM}$ & $\mathrm{PS}$ & $\mathrm{ZO}$ & $\mathrm{NM}$ & $\mathrm{PM}$ & $\mathrm{PB}$ & $\mathrm{PB}$ \\
$\mathrm{PB}$ & $\mathrm{NS}$ & $\mathrm{NM}$ & $\mathrm{NB}$ & $\mathrm{NB}$ & $\mathrm{PB}$ & $\mathrm{PB}$ & $\mathrm{PB}$ \\
\hline
\end{tabular}

\section{Discussion AND RESULTS}

According to real following situations, the experiments assume that vehicles are traveling in ideal status, intensive status and dangerous status, and compare the changes of real distance in different statuses. Automatic control theory suggests that the ideal changing curve obtained by the controller should be smooth and eventually converging to the safe distance. Similarly, response time, overshoot, and stable time are also significant for evaluating control effect. With the requirements of different situations, the corresponding control parameters should be adjusted in real time. In the simulation, PID parameters are initialized $\mathrm{k}_{\mathrm{p}}=20, \mathrm{k}_{\mathrm{i}}=0.5, \mathrm{k}_{\mathrm{d}}=1$. According to the rules of traffic, the initial speed of vehicle $\mathrm{F}$ and $H$ are $80 \mathrm{~km} / \mathrm{h}, 100 \mathrm{~km} / \mathrm{h}$. The distances are set as $121 \mathrm{~m}$, $72 \mathrm{~m}, 53 \mathrm{~m}$ in ideal status, intensive status and dangerous status, respectively.

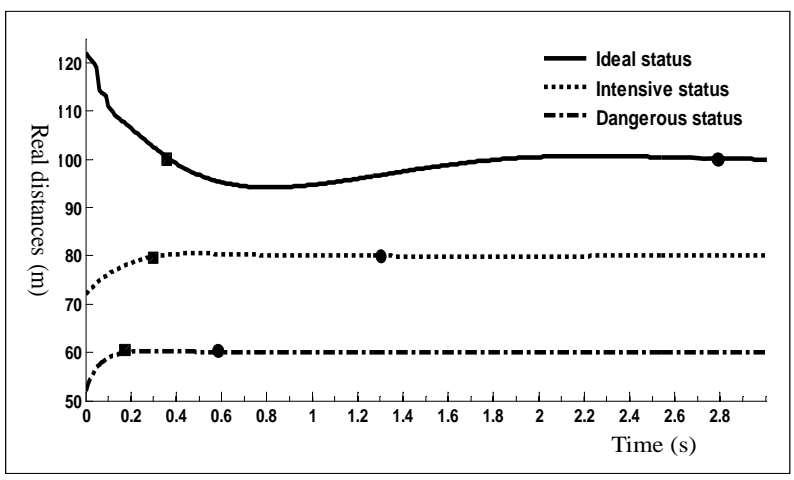

Figure 6. Changes of real distances between vehicles in different driving statuses.

Fig.6 shows the effects of three different driving statuses obtained by fuzzy PID controller. From top to bottom, the curves represent different changes of real distances in ideal status, intensive status and dangerous status, and the current safe distances are $100 \mathrm{~m}, 80 \mathrm{~m}$ and $60 \mathrm{~m}$, respectively. As is shown in Fig.6, the overshoot of adjustment in ideal status is about $10 \mathrm{~m}$, and the response time of system is slowest, about $3 s$. Though the adjustment of the process is relatively slow, it ensures comfortable driving, and eventually stabilizes at safe distance, which properly meets the demands of driving in ideal status. For the intensive status, response time of the system and the corresponding stable time are advanced $0.2 \mathrm{~s}$ and $2 \mathrm{~s}$ at least. Especially in dangerous status, response time is less than $0.2 \mathrm{~s}$, and the object adjusts itself within $0.4 \mathrm{~s}$, which greatly improves dynamic performance of the object and efficiently decreases risk of collision.

In order to evaluate the competence of the proposed control system based on fuzzy PID controller, another control system on the basis of general PID was designed. It is shows the results of comparison under the controls of fuzzy PID and general PID in Fig.7. In this experiment, three different statuses, ideal status, intensive status and dangerous status, are all conducted to evaluate the effect of fuzzy PID controller, as (a), (b), (c). The speed of the vehicles and parameters of controller are all initialized same as the above. The distances from the vehicles ahead are set as $122 \mathrm{~m}, 72 \mathrm{~m}$ and $43 \mathrm{~m}$ in different situations.

The illustration shows that the response time, overshoot and stable time are all improved to varying degrees for the demands of different statuses. In ideal status, the effect of adjustment is not very obvious, but the small change of overshoot ensures a comfortable driving. It is modest to control the object in intensive status, which is helpful to drive cautiously. Especially adjusting in dangerous status, response time and stable time are decreased substantially. Comparing with the general PID, the overshoot is reduced $3 \mathrm{~m}$, which is greatly significant for increasing the possibilities of safe driving in dangerous status. Therefore, as the results of simulations mentioned, the self-optimizing PID model based on fuzzy control is proved to be significant and efficient for improving dynamic abilities of the system and ensures the safety driving of vehicles in different situations.

\section{CONCLUSION}

This paper proposes a self-optimizing PID model based on fuzzy control, which not only inherits advantages of general PID control, but also combines characteristics of fuzzy control for flexible control. The simulation results show that vehicles can follow safe distance and get faster response time for providing reliable protection by the proposed model. Moreover, the changes of speed, acceleration and other factors are also important for impacting studies of following, the safety control model will be further improved with more researches. 


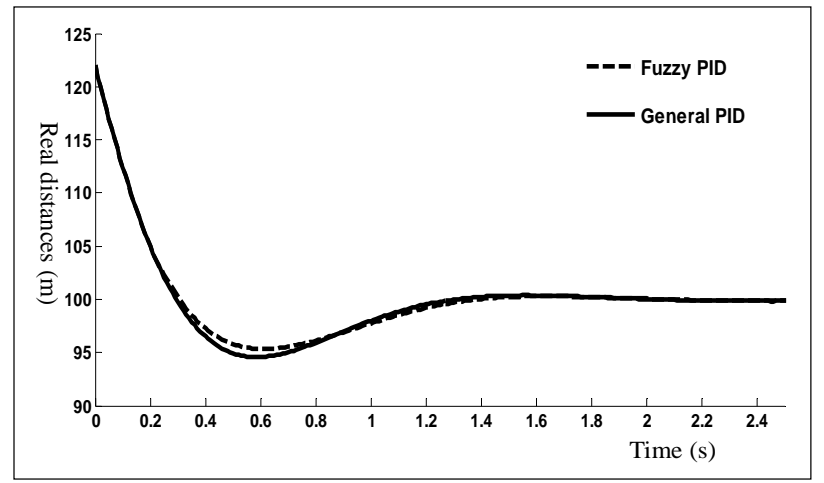

(a)

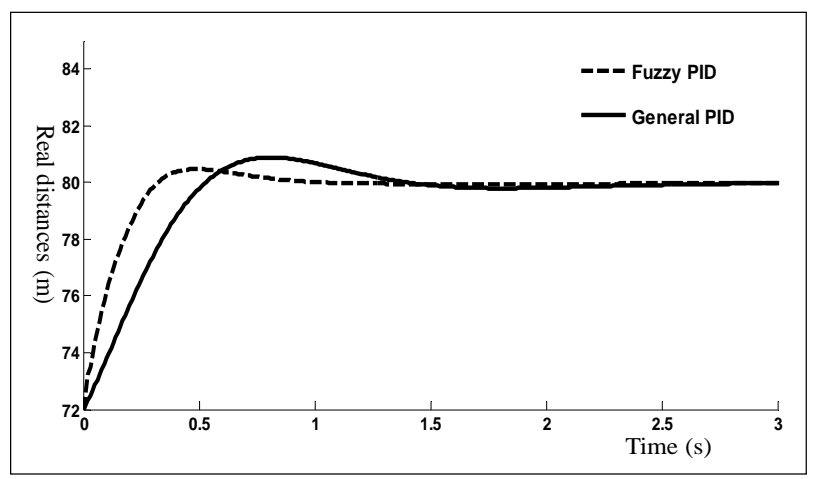

(b)

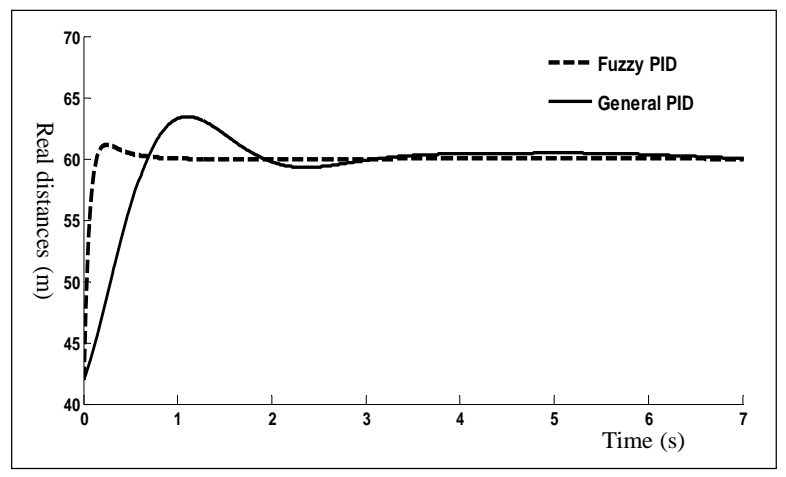

(c)

Figure 7. Changes of real distances between vehicles under different control modes (a) vehicles in ideal status(b) vehicles in intensive status (c) vehicles in dangerous status.

\section{ACKNOWLEDGMENT}

This work was supported by the Natural Science Foundation of China (61174177 and 11171079).

\section{REFERENCES}

[1] Weigao Qiao, and Bo Shang, "Current situation analysis and safety countermeasure study on road traffic accidents in China," International Conference on Remote Sensing, Environment and Transportation Engineering, pp. 3034 - 3037, June 2011.

[2] Ministry of Transport of the People's Republic of China, http://www.moc.gov.cn/zhuzhan/jiaotongguihua.

[3] Xiaoyuan Wang, and Xinyue Yang, "Review of the simulation model of driving behavior," Proceedings of the Fifth International Conference on Machine Learning and Cybernetics, pp. 13-16, August 2006.

[4] F. Jimenez, F. Aparicio, and J. Paez, "Evaluation of in-vehicle dynamic speed assistance in Spain: Algorithm and driver behavior,” IET Intell. Transp. Syst., Vol. 2, pp. 132 -142, June 2008.

[5] E. Adell, A. V\&aacute rhelyi, M. Alonso, and J. Plaza, "Developing HMI components for a driver assistance system for safe speed and safe distance,” IET Intell. Transp. Syst., vol. 2, pp. 1 -14, March 2008.

[6] A. Amditis, E. Bertolazzi, M. Bimpas, F. Biral, P. Bosetti, M. Da Lio, et al. "A holistic approach to the integration of safety applications: The INSAFES subproject within the European framework programme 6 integrating project PReVENT,” IEEE Trans. Intell. Transp. Syst., vol. 11, pp. 554 -566, September 2010.

[7] Khodayari A, Ghaffari A, Nouri M, Salehinia S, and Alimardani F, "Model Predictive Control system design for car-following behavior in real traffic flow," IEEE International Conference on Vehicular Electronics and Safety, pp. 87-92, July 2012.

[8] E. Bertolazzi, Biral F, Da Lio M, Saroldi A, and Tango F, “Supporting drivers in keeping safe speed and safe distance: The saspence subproject within the European framework programme 6 integrating project PReVENT,” IEEE Trans. Intell. Transp. Syst., vol. 11, pp. 525 -538, September 2010.

[9] Samad Kamal, and M.A, "Smart Driving of a Vehicle Using Model Predictive Control for Improving Traffic Flow," IEEE Transactions on Intelligent Transportation Systems, vol. 15, pp. 878-888, April 2014.

[10] Gouy M, Diels C, Reed N, Stevens A, and Burnett G, "Do drivers reduce their headway to a lead vehicle because of the presence of platoons in traffic? A conformity study conducted within a simulator," Intelligent Transport Systems, vol. 7, pp. 230 - 235, June 2013.

[11] Korkmaz M, Aydogdu O, Dogan H, "Design and performance comparison of variable parameter nonlinear PID controller and genetic algorithm based PID controller” International Symposium on Innovations in Intelligent Systems and Applications (INISTA), pp. 1-5, July 2012.

[12] Lilong Gong, Lihua Luo, Hui Wang, and Hong Liu, "Adaptive Cruise Control Design Based on Fuzzy-PID,” International Conference on EProduct E-Service and E-Entertainment (ICEEE), pp. 1-4, November 2010.

[13] Talebi Abatari H, and Dehghani Tafi A, "Using a Fuzzy PID Controller for the Path Following of a Car-like Mobile Robot,” International Conference on Robotics and Mechatronics, pp. 189-193, February 2013.

[14] Alonso L, Perez-Oria J, Al-Hadithi B.M, and Jimenez A, "Self-Tuning PID Controller for Autonomous Car Tracking in Urban Traffic," The 17th International Conference on System Theory, Control and Computing (ICSTCC), pp. 15-20, October 2013. 